\title{
Robust combining methods in committee neural networks
}

\begin{abstract}
Combining a set of suitable experts can improve the generalization performance of the group when compared to single experts alone. The classical problem in this area is to answer the question about how to combine the ensemble members or the individuals. Different methods for combining the outputs of the experts in a committee machine (ensemble) are reported in the literature. The popular method to determine the error in every prediction is Mean Square Error (MSE), which is heavily influenced by outliers that can be found in many real data such as geosciences data. In this paper we introduce Robust Committee Neural Networks (RCNNs). Our proposed approach is the Huber and Bisquare function to determine the error between measured and predicted value which is less influenced by outliers. Therefore, we have used a Genetic Algorithm (GA) method to combine the individuals with the Huber and Bisquare as the fitness functions. The results show that the Root Mean Square Error (RMSE) and R-square values for these two functions are improved compared to the MSE as the fitness function and the proposed combiner outperformed other five existing training algorithms.
\end{abstract}

Keyword: Committee machine; Neural network; Genetic algorithms; Bisquare and Huber function 